\title{
Thermal Inspection of a Composite Fuselage Section using a Fixed Eigenvector Principal Component Analysis Method
}

\author{
Joseph N. Zalameda*a ${ }^{*}$ Sean Bolduc ${ }^{\mathrm{b}}$, and Rebecca Harman ${ }^{\mathrm{b}}$, \\ aNASA Langley Research Center Hampton, VA 23681-2199; \\ bASA Internships, Fellowships and Scholarships (NIFS) Langley Research Center Hampton, VA \\ 23681-2199
}

\begin{abstract}
A composite fuselage aircraft forward section was inspected with flash thermography. The fuselage section is 24 feet long and approximately 8 feet in diameter. The structure is primarily configured with a composite sandwich structure of carbon fiber face sheets with a Nomex ${ }^{\circledR}$ honeycomb core. The outer surface area was inspected. The thermal data consisted of 477 data sets totaling in size of over 227 Gigabytes. Principal component analysis (PCA) was used to process the data sets for substructure and defect detection. A fixed eigenvector approach using a global covariance matrix was used and compared to a varying eigenvector approach. The fixed eigenvector approach was demonstrated to be a practical analysis method for the detection and interpretation of various defects such as paint thickness variation, possible water intrusion damage, and delamination damage. In addition, inspection considerations are discussed including coordinate system layout, manipulation of the fuselage section, and the manual scanning technique used for full coverage.
\end{abstract}

Keywords: Thermal nondestructive evaluation, large area inspection, composite fuselage, water intrusion damage, delamination, composite sandwich structure

\section{INTRODUCTION}

A composite fuselage aircraft forward and aft sections were obtained by the nondestructive evaluation sciences branch (NESB) NASA Langley for nondestructive evaluation (NDE) measurements. The aircraft sections were manufactured around 2004 and were obtained from Wichita State University. The aircraft sections were from a Hawker 4000, a midsize business jet manufactured by Beechcraft. This jet represented the first in its class to primarily utilize a composite construction. The fuselage forward section is 7.3 meters long and approximately 2.4 meters in diameter. Pictures of the fuselage sections are shown in figure 1. The composite aircraft is comprised of a composite sandwich structure with both Nomex ${ }^{\circledR}$ and aluminum core. The outer skins were carbon fiber face sheets. Flash thermography was used to perform full coverage inspection and has been shown to be an effective inspection technique for composite

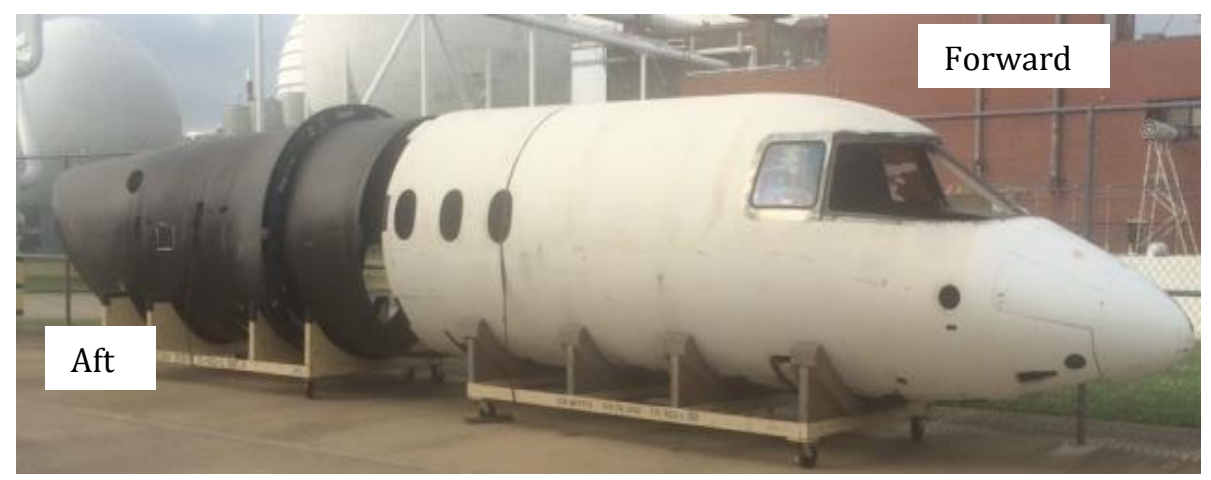

Figure 1. Forward and aft fuselage sections.

*joseph.n.zalameda@nasa.gov; phone 1757-864-4793; fax 1 757-864-4914; http://nde.larc.nasa.gov 
sandwich structures [1-4]. Flash thermography advantages are noncontact, rapid and safe. The inspection requires only a small amount of heat (less than 10 degrees $\mathrm{C}$ above ambient). The outer mold line (OML) area of the forward section was inspected. The flash thermography inspection foot print was approximately $23 \times 33$ centimeters. Each inspection footprint represented a thermal data set to be processed into an inspection image. For full coverage, the thermal data consisted of 477 data sets totaling in size of over 227 Gigabytes and covering approximately 36 square meters. Principal component analysis (PCA) was used to process the data sets for substructure and defect detection. A fixed eigenvector approach using a global covariance matrix was used and compared to a varying eigenvector approach. The fixed eigenvector approach was demonstrated to be a practical analysis method for the detection and interpretation of various defects such as paint thickness variation, possible water intrusion damage, and delamination damage. In addition, inspection considerations are discussed including coordinate system layout, manipulation of the fuselage section, and the manual scanning technique used for full coverage.

\section{FUSELAGE INSPECTION}

A commercially available flash thermography system was used for the inspection. The flash thermography system consisted of two linear flash tubes mounted within a hood. An infrared camera was mounted at the back of the hood viewing through a circular hole between the flash tubes and were positioned to view the hood opening. In this configuration, the flash lamps heated an area equal to the hood opening and the infrared camera captured the thermal response. The infrared camera operates in the mid-wave infrared band (3-5 micrometers) and is configured with a 25 $\mathrm{mm}$ germanium lens. The focal plane array size for the camera is $1344 \times 784$ with a detector pitch size of $14 \times 14$ micrometers. The inspection area was approximately $23 \times 33$ centimeters. The camera frame rate was $60 \mathrm{~Hz}$. For each inspection, 10 frames were acquired before heating and 890 frames were acquired after the flash (14.83 seconds). For full coverage, a coordinate system was necessary to track the data sets corresponding to the location on the fuselage. The coordinate system was started on the crown starting with the letter A and ending with the letter $\mathrm{O}$ on both the starboard and port sides. This defined each row around the circumference. The columns within each row were designated by a number from 1 to 20 starting from the fuselage front (nose). Not every row had 20 columns because of doors, windows, and other features that lacked the composite structure of interest. Two areas on the tapering nose did not fit the regular row-column layout, and these were labeled FS and FP for front starboard and front port, respectively. Small lead dots that were approximately $3 \mathrm{~mm}$ in diameter were placed in the four corners of each area. These dots could easily be seen on the IR imager when collecting data, which ensured that the entire area under inspection was being captured and facilitated the alignment of thermal images to form a mosaic over the fuselage. The thermal hood was mounted onto an extension boom as shown in figure 2. The extension boom was manipulated manually. To access the top and bottom areas, the fuselage was rotated using an overhead crane.

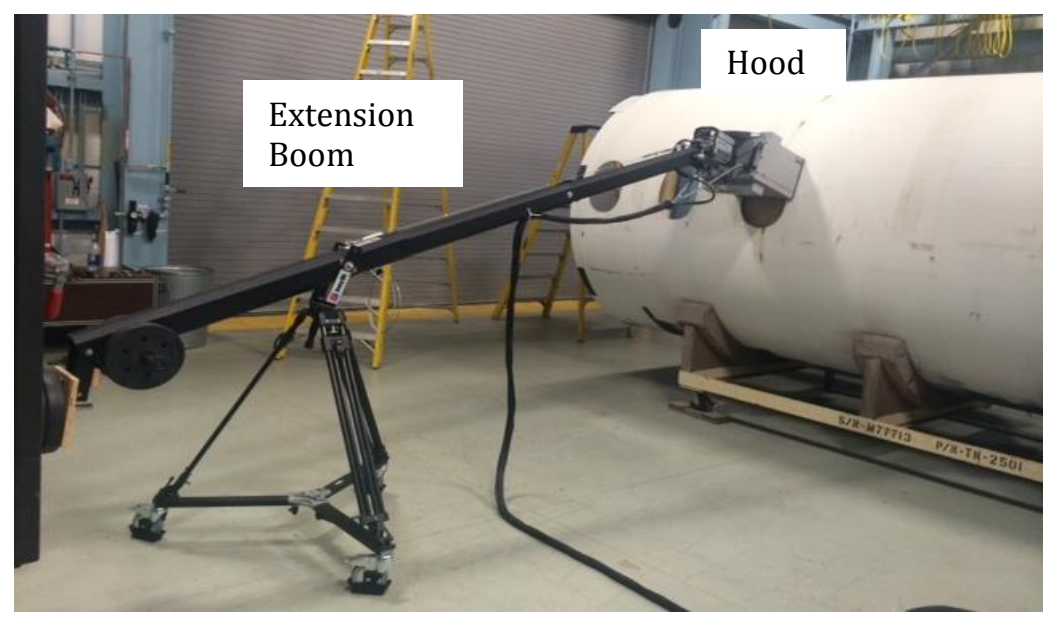

Figure 2. Thermal inspection setup for forward section measurements. 


\section{PRINCIPAL COMPONENT ANALYSIS}

Principal component analysis (PCA) is common for processing of thermal data [5,6]. This algorithm is based on decomposition of the thermal data into its principal components or eigenvectors. Singular value decomposition is a routine used to find the singular values and corresponding eigenvectors of a matrix. Since thermal NDE signals are slowly decaying waveforms, the predominant variations of the entire data set are usually contained in the first or second eigenvectors, and thus account for most of the data variance of interest. The principle components are computed by defining a data matrix A, for each data set, where the time variations are along the columns and the spatial image pixel points are row-wise. The matrix $\mathrm{A}$ is adjusted by dividing the maximum value (normalization) and subtracting the mean along the time dimension. The covariance matrix is defined as the $A^{T * A}$. The covariance matrix is now a square matrix of number of images used for processing. The covariance matrix can then be decomposed using singular value decomposition as:

$$
\text { covariance matrix }=A^{T} A=U * S * U^{T}
$$

where $S$ is a diagonal matrix containing the squares of the singular values and $U$ is an orthogonal matrix, which contains the basis functions or eigenvectors describing the time variations. The eigenvectors can be obtained from the columns of $\mathrm{U}$. The PCA inspection image is calculated by dot product multiplication of the selected eigenvector times the temperature response (data matrix A), pixel by pixel.

\subsection{Local Eigenvector Calculation}

For each data set using region of interest pixels of 514 x 369, local eigenvectors are calculated for their respective PCA inspection images. Shown in figure 3 are eigenvectors calculated for a typical thermal data set (starboard side row I column 4). A processing time window of 1.5 to 4.83 seconds was used (200 frames). A time delay of 1.5 seconds allowed enough time for the heat to flow through the composite face sheet and into the core and 4.83 seconds was sufficient time to provide good contrast of the underlying geometry [4]. Some thicker laminate regions around the door and window frames would require longer time windows, however for this study only sandwich structure was of interest. For this time window the thermal images were processed using principal component analysis to determine the eigenvalues and respective eigenvectors.

Eigenvector \#1 has a slow decay, dominated by the transient cool down after the flash and corresponds to an eigenvalue of 2,076.4. This represents the dominate variance in the data set. Eigenvector \#2 corresponds to an eigenvalue of 2.67 and is less dominate. This defines the contrast variance from substructure that is more deeply buried within the composite with a maximum of around 2.75 seconds after the flash. Eigenvector \#3 and \#4 correspond to eigenvalues 0.38 and 0.13 respectively. As shown in figure 3, these eigenvectors are more influenced by noise and thus result in images that have less spatial detail of interest. The first or second eigenvector PCA image provides good contrast for defect detection [4]. This is shown in figure 4 where the processed images from 3 data sets, starboard side Row I columns: 4, 5, and 6 are assembled to correspond to the visible image. The eigenvector \#2 images clearly show the substructure as compared to eigenvector \#1, \#3, and \#4 images.

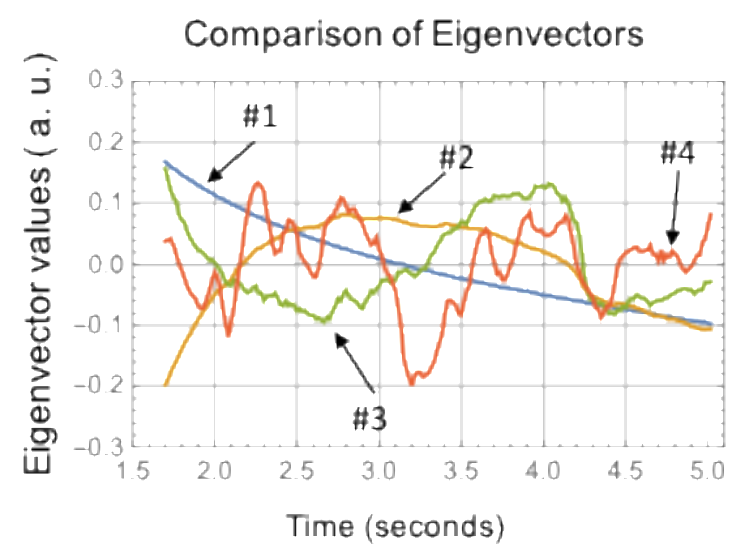

Figure 3. Comparison of eigenvectors 1 through 4. 


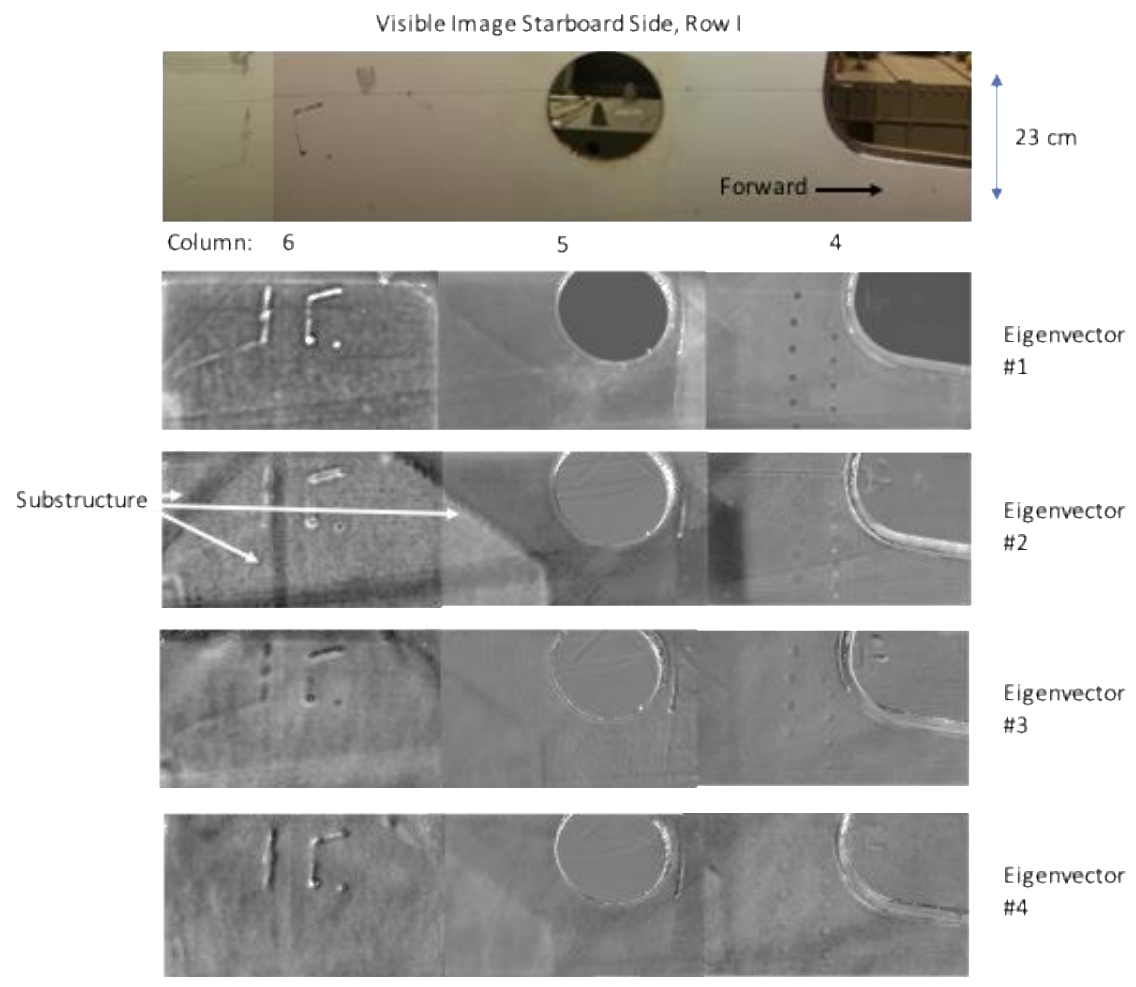

Figure 4. Comparison of eigenvectors \#1 through \#4 processed imagery with eigenvector \#2 showing good underlying contrast.

\subsection{Fixed Eigenvector Calculation}

To facilitate comparison of inspection images a fixed eigenvector processing approach is used [6]. The fixed eigenvector processing approach utilizes an averaged covariance matrix from all the data sets. This is given in equation 2 where $i$ is the data set number, $N$ is the total number of data sets, and $A_{\mathrm{i}}$ is the respective data matrix.

$$
\text { averaged covariance matrix }=\frac{1}{N} \sum_{i=1}^{N} A_{i}^{T} A_{i}
$$

From the averaged covariance matrix a single set of eigenvectors are calculated. The selected eigenvector, in our case eigenvector \#2, is then used to process all the data sets by matrix multiplication of the fixed eigenvector with the data matrix A for a given data set. This assures the pixel grayscale values are consistent for similar material, underlying geometry, or defect. This also provides grayscale uniformity for the assembled images into one larger mosaic inspection image. This can be seen in figure 5 where the processed images from 3 data sets, starboard side Row I columns: 4, 5, and 6 are assembled. The assembled image grayscale values are not consistent from image to image for the local eigenvector calculation. These areas are marked with white circles in figure 5. The bottom row of images in figure 5 are calculated using a fixed eigenvector. These are more uniform in gray scale from image to image and helps to determine consistent underlying geometry or identify defect areas. This can be seen in figure 6 where the inspection images for the entire data set are assembled into one mosaic. This mosaic consists of 477 processed data sets using a fixed eigenvector resulting in 477 inspection images. The curved surfaces made assembling the images difficult especially as the diameter tapered toward the nose. Nonetheless, the overall 2-D image provides a good inspection of the OML fuselage forward section. The diagonal darker lines appear to be core spliced areas where core sections are attached using a foam adhesive filler with perhaps ply overlaps for reinforcement. Shown in figure 6 is the inspection area: port side, row $\mathrm{n}$ column 17 that was erroneously omitted during data collection. An automated robotic system would be more appropriate to ensure entire coverage. 


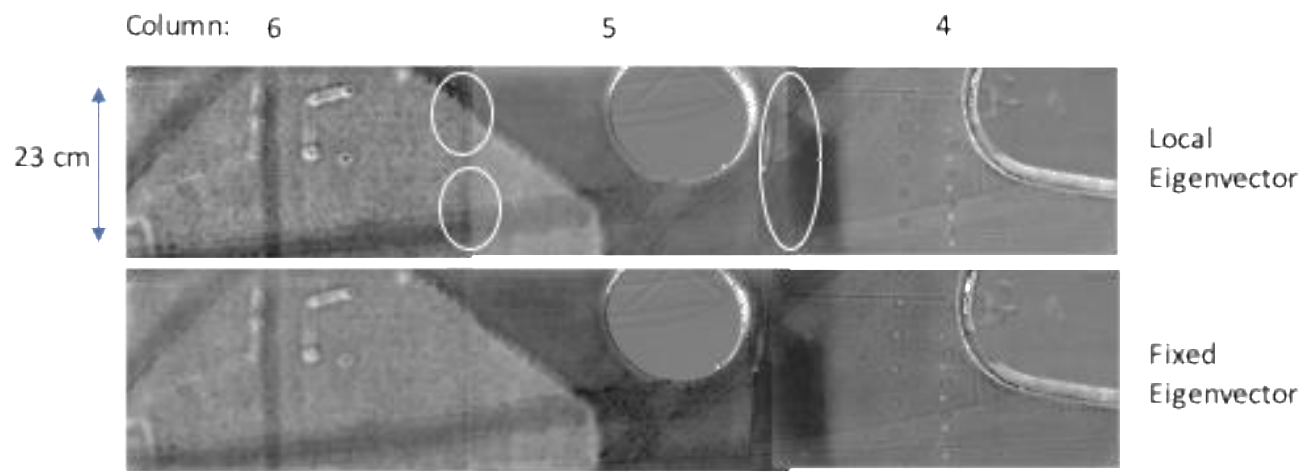

Figure 5. Comparison of local to fixed eigenvectors and note uniform grayscale in fixed eigenvector image.

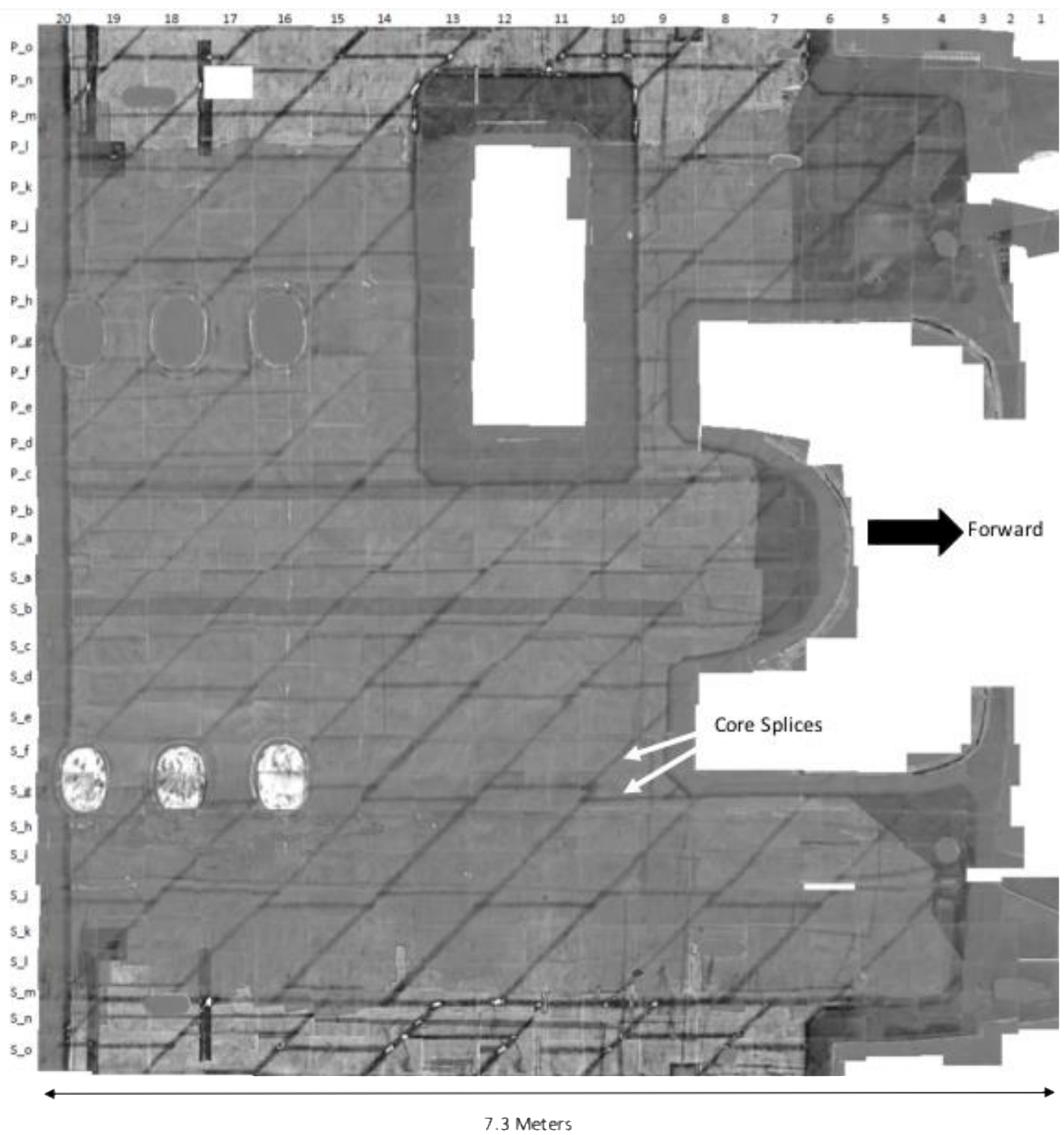

Figure 6. Entire data set assembled into one mosaic. This mosaic consists of 477 data sets processed using a fixed eigenvector. 


\section{DEFECT DETECTION}

The fuselage section has surface visible damage in the form of deep scratches, gouges, and discolored areas. In addition, it was found through handling that water intrusion (water droplets leaking out when rotating fuselage) and crushed core damage was also evident. For some of these defects thermography was effective in determining the extent of the damage. Example inspection images of surface scratches/scuff marks, discoloration, and a gouge is shown in figure 7. The thermal inspection images reveal that the damage shape is very similar to the visible imagery. This indicates the damage is localized to the visible shape. This is also true for the gouge defect shown in the bottom right image of figure 7. The discolored area shown in the bottom left image of figure 7 is a rust spot. This rust spot appears to be rusted metal (from a metal support) that has adhered to the outer surface. Without the visible image the corresponding thermal inspection image would appear to show a defect at the rust spot location. From figure 7, one can conclude that the visible imagery is important to interpret the thermal inspection images. Additionally, the areas of the fuselage underside were not painted and this was enough to change the thermal response as shown in figure 8 . This shows the sensitivity of the thermal inspection to "see through the paint" and it appears the paint did not limit the inspection of the fuselage.

Visible Image Port Side, Row $\mathrm{H}, \mathrm{Col} 13$ Thermal Inspection Image

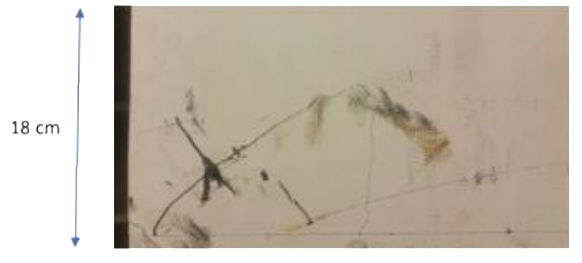

Visible Image Port Side, Row H, Col 16

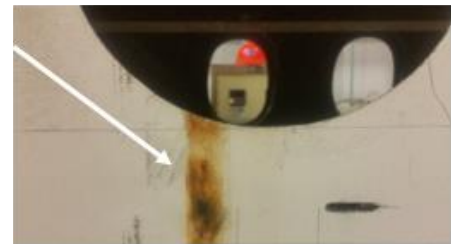

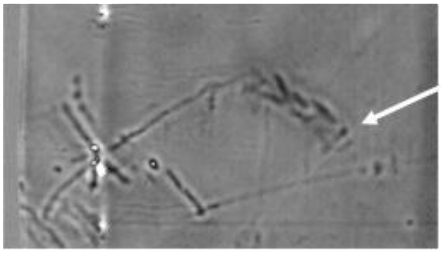

Thermal Inspection Image

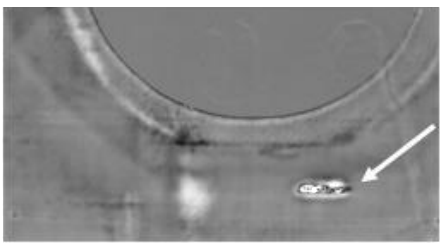

Scratches

Scuffs

Gouge

Figure 7. Example inspected areas of surface scratches, gouges, and discoloration.

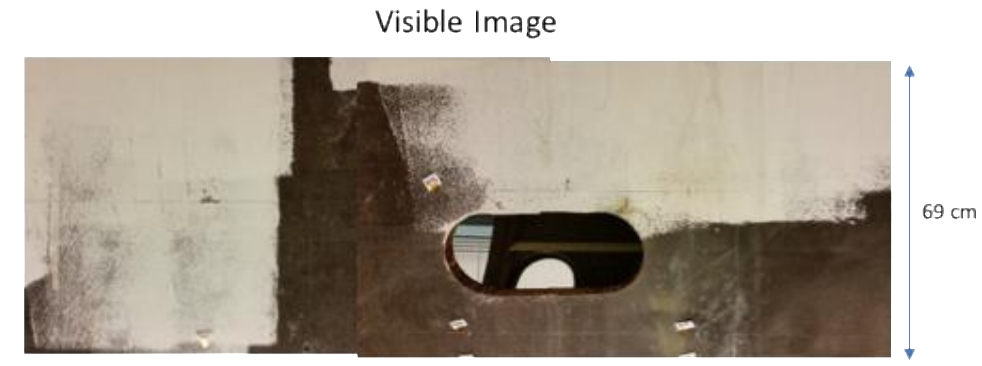

Thermal Inspection Image

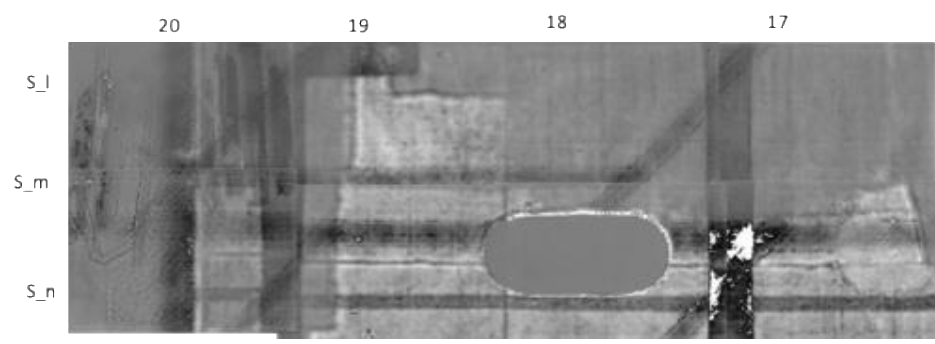

Figure 8. Variation in the thermal inspection imagery due to paint. 
A crown section adjacent to the cockpit window contained a region of core crushed (larger than the inspection footprint). The face sheets in these regions could be compressed to over $1.27 \mathrm{~cm}$ by merely pressing on them. The flash thermal inspection was not able to detect the core crushed region as shown in figure 9. The damaged core does not affect thermal response because the Nomex ${ }^{\circledR}$ core thermal properties are very close to those of air. Lastly, suspected water damaged areas were detected just below the starboard side windows. It appears water may have migrated from the window edge into the fuselage. Water damage is suspected because the fuselage section was exposed to the weather, some water was noted dripping (very small amount) from the fuselage during rotation, and also the irregular shape of the suspected water damage from the thermal inspection images as shown in figure 10. Sectioning the fuselage would help to determine if this is actual water damage. Also of interest is what underlying change in the thermal properties of the substructure would cause the irregular shape detected in figure 10. Similar suspected water damage areas were also detected adjacent to the door bottom. No passive cool spots were detected that would indicate water evaporation during thermal data collection.

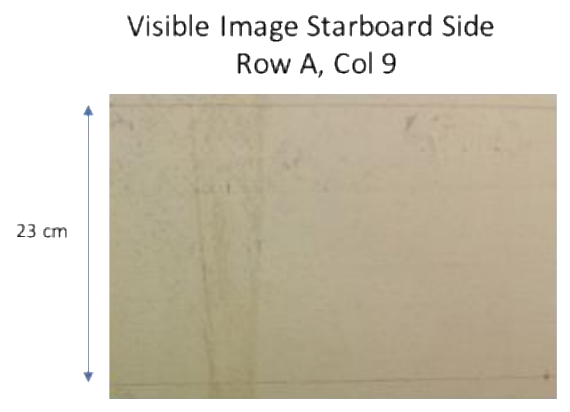

Thermal Inspection Image

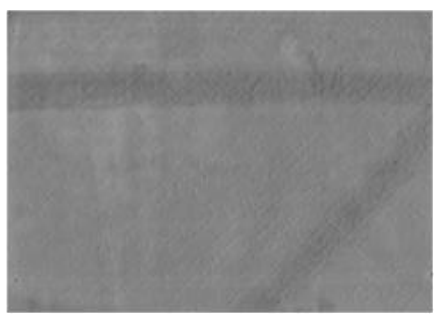

Figure 9. Thermal inspection of core crushed area showing no obvious thermal indication.

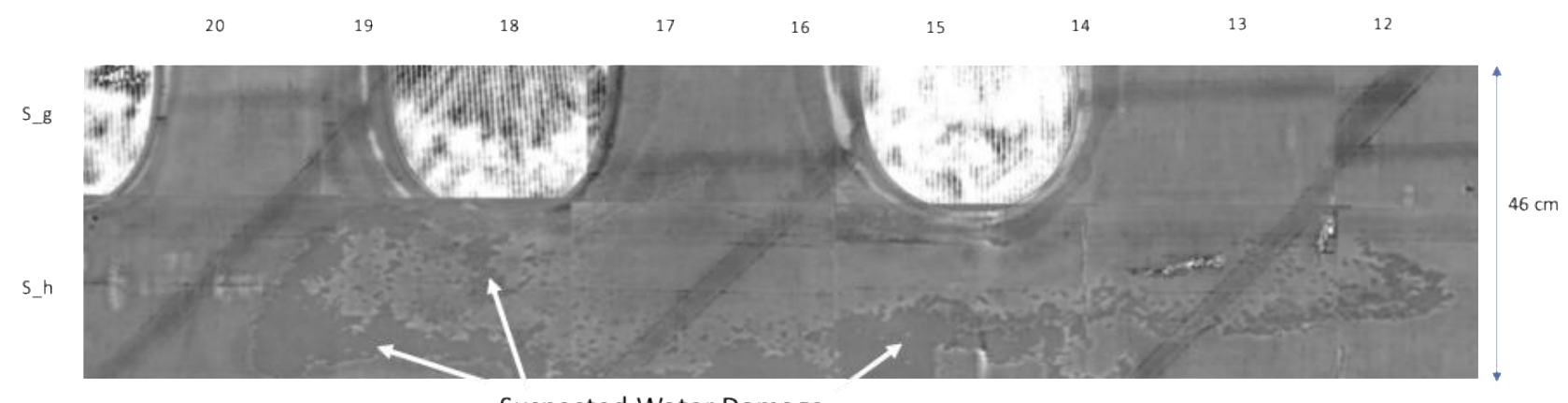

Suspected Water Damage

Figure 10. Thermal inspection of suspected water damaged areas.

\section{CONCLUSIONS}

A fixed eigenvector approach using a global covariance matrix was used to inspect the outer area of a forward composite fuselage aircraft section covering approximately 36 square meters. The fixed eigenvector approach was demonstrated to be a practical analysis method for the detection and interpretation of various defects such as paint thickness variation, possible water intrusion damage, and delamination damage. Comparison of these results to destructive testing would help to validate the thermal measurements. Also if the fuselage was sectioned into smaller samples then perhaps X-ray CT would help validate the suspect water damage areas. The manual manipulation of the inspection hood and rotation of the fuselage required approximately 2 work weeks ( 80 hours) to collect the data. An automated robotic inspection would be faster and insure full coverage. 


\section{REFERENCES}

[1] Sfarra, S. et al, "A comparative investigation for the nondestructive testing of honeycomb structures by holographic interferometry and infrared thermography “,J. Phys.: Conf. Ser. 214 012071, (2010).

[2] Clemente Ibarra-Castrando, et al, "Comparative Study of Active Thermography Techniques for the Nondestructive Evaluation of Honeycomb Structures", Research in Nondestructive Evaluation Volume 20, Issue 1, (2009).

[3] Genest, M., Brothers, M., Beltempo, C. A., Rutledge, R. S., "Nondestructive Evaluation of Aluminum Honeycomb Sandwich Structures", NATO Unclassified, STO-MP-AVT-224, (2013).

[4] Zalameda, J. and Raymond, P., "Thermal inspection of composite honeycomb structures", edited by Stockton, Colbert, and Hsieh, Proc. SPIE 9105, Thermosense: Thermal Infrared Applications XXXVI, 91050C (2014).

[5] Rajic, N., "Principal Component Thermography for Flaw Contrast Enhancement and Flaw Depth Characterisation in Composite Structures", Composite Structures, Vol. 58, pp. 521--528, (2002).

[6] Cramer, K. E. and Winfree, W. P., "Fixed Eigenvector Analysis of Thermographic NDE Data", Proceedings of SPIE, Thermosense XXXIII, edited by Morteza Safai and Jeff Brown, Vol. 8013, (2011). 